\title{
A Comprehensive Morphological Hybrid Filter Considering Symmetry Maintained
}

\author{
Yuefeng $\mathrm{Lu}^{1}$, Shaofeng $\mathrm{Lu}^{2, *}$, Baomin $\mathrm{Han}^{1}$, Jianhua $\mathrm{Wu}^{3}$, XiZhi Chang ${ }^{4}$, Chen \\ Feng $^{1}$, Shuo Liu ${ }^{1}$ \\ ${ }^{1}$ School of Civil and Architectural Engineering, Shandong University of \\ Technology, Zibo 255049, China \\ ${ }^{2}$ College of Information Science and Engineering, Northeastern University, \\ Shenyang 110819, China \\ ${ }^{3}$ School of Geography and Environment, Jiangxi Normal University, Nanchang \\ 330022, China \\ ${ }^{4}$ School of Materials Science and Engineering, Shandong University of \\ Technology, Zibo 255049, China \\ lushaofeng21@163.com,yflu@sdut.eduvcn
}

\begin{abstract}
In this paper, we present a comprehensive morphological hybrld filter considering symmetry maintained, based on composited the morphological hybrid filters and the generalized morphological hybrid filters. The filter improved filtering performance from two aspects: morphological operate mode and optimization of structure elements. It can eliminate the gray values deviation of the images und have the double advantage of the two-path dual filters. Simulation results show that the new hybrid filter can effectively remove the noise in the image while maintaining the image details. Compared with the other morphological hybridfilters, the imagesfiltered by the new filter may have a higher peak signal-to-noise ratio (PSNR) and a maller mean square error (MSE).
\end{abstract}

Keywords: mathenatical morphology; generalized morphological filters; hybrid filters; symmetry maintained; Goussian noise; PSNR

\section{Introdaction}

Mathematical morphology is a new theoretical discipline which based on integral geometry and random set theory, it uses a set of transformation,operation,conception and algorithm to describe the basic features or the basic structure of images, and it is an effective tool on the nonlinear morphological analysis and description[1,2].Mathematical morphology is widely used in all aspects of the image processing, including image preprocessing[3,4],edge detection[5,6,7,8], feature extraction[9,10] and breakpoints connection, and so on.

\footnotetext{
Morphological filter is a nonlinear filter based on mathematical morphology, its basic idea is using structural elements to probe the image, preserving structures that consistented with structural elements and removing that not consistented with(such as noise,glitch,and so on),maintaining more image details while filtering out noise[11,12].The basic morphological filter includes morphological opening filter, morphological closing filter, morphological opening-closing filter, morphological closingopening filter[1,13,14],these filters apply the same structural elements, maintaining a part of details and at the same time possibly filtering out a part of useful details. So there comes generalized morphological opening filter, generalized morphological closing filter, generalized morphological opening-closing filter, generalized morphological closingopening filter[11,15,16], but these morphological filters will result in grey values deviation
} 
of the images after being filtered whether started with opening filter or closing filter. So the morphological hybrid filter and the generalized morphological hybrid filter that combine opening operation and closing operation in cascade come into being $[8,16,17]$,and these hybrid filters have better filtering effect.

Aiming at grey values deviation of the images after being filtered by traditional morphological filters, Lei [17] analyzed five kinds of morphological hybrid filters, it turns out that the basic morphological opening and closing hybrid filter and the generalized morphological opening and closing hybrid filter have an advantage over other hybrid filters in terms of the capability of suppressing noise, symmetry and time consuming. It also points out that the advantage of hybrid filters lies in it does not depend on filters whether started with opening filter or closing filter, and it has the dual advantages of the two-path dual filter, therefore, this paper combines morphological hybrid filters and generalized morphological filters to propose a kind of comprehensive morphological hybrid filter considering maintaining symmetry. The result of simulation experiment shows that the new hybrid filter can not only remove the noise in the image effectively, but also preserve the image details. Compared with the other morphological hybrid filters, the images filtered by the new filter may have a higher peak signal-to-noise ratio (PSNR) and a smaller mean square error (MSE).

\section{Morphological Filter}

\subsection{Basic Morphological Filter}

We define $f(x, y)$ and $k(x, y)$ as two discrete functions that based on two-dimensional discrete space, $f(x, y)$ is the grey value function of in phtted image at point $(x, y), k(x, y)$ is the grey value function of structural elements at foint $(x, y), D_{f}$ and $D_{k}$ are the domains of $f(x, y)$ and $k(x, y)$ respectively. The definitions of grey expansion and grey erosion are:

$f \oplus k(x, y)=\max _{(s, t)=D_{k}}\{f(x-s, y-t)+k(s, t)\}$

$f \oplus k(x, y)=\max _{(s, t)=D_{k}}\{f(x-s, y-t)+k(s, t)\}$

So the definitions of morphological opening filter and morphological closing filter are $[13,14]$ :

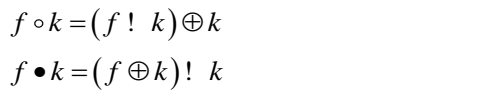

Because morphological opening filters can suppress positive pulse noise(that is peak noise) in signal, so if can eliminate peak area that smaller than the selected structural elements and at tha same time it can not only keep the overall image grey values unchanged but also do not substantially affect the big peak area. But because morphological closing filters can suppress negative pulse noise(that is bottom noise) in signal, o it ean eliminate bottom area that smaller than the selected structural elements while $\mathrm{t}$ ean not only keep the overall image grey values unchanged but also do not substantially affect the big bottom area. Making use of the complementary nature of opening operation and closing operation on suppressing noise to combine opening operation and closing operation in cascade, the morphological opening-closing filter and morphological closing-opening filter can suppress positive pulse noise and negative pulse noise in signal simultaneously. The common morphological filters that based on combining opening operation and closing operation in cascade are (in order to represent conveniently, we use $\mathrm{O}$ as opening operation and $\mathrm{C}$ as closing operation):

$\mathrm{OC}=f \circ k \bullet k$

$\mathrm{CO}=f \bullet k \circ k$ 


\subsection{Generalized Morphological Filter}

Since the basic morphological filters all use the same structural elements, these filters can only maintain one kind of geometric information but filter out others, it is of no benefit to maintain image details. Aiming at the basic morphological filters, many scholars come up with generalized morphological opening filters, generalized morphological closing filters, generalized morphological opening-closing filters and generalized morphological closing-opening filters [11, 15, and 16].These filters are made of different sizes of structural elements and they can preserve the image details in the process of filtering out noise. Here are definitions of the four kinds of generalized morphological filters (we use $\mathrm{G}$ as generalized):

$$
\begin{aligned}
& \mathrm{GO}=\left(f ! k_{1}\right) \oplus k_{2} \\
& \mathrm{GC}=\left(f \oplus k_{1}\right) ! k_{2} \\
& \mathrm{GOC}=f \circ k_{1} \bullet k_{2} \\
& \mathrm{GCO}=f \bullet k_{1} \circ k_{2}
\end{aligned}
$$

$k_{1}, k_{2}$ are two different structural elements and $k_{1} \subseteq k_{2}$.

\subsection{Morphological Hybrid Filter}

As we can see from the definitions of opening fitters closing filters, opening-closing filters and closing-opening filters, opening-closing filters or closing-opening filters are started with erosion or expansion to filter images essentially. Through the properties of erosion and expansion we can know that the inage grey values will be down (darker than the original image) or up (brighter than the original image) in different degrees after being filtered by opening-closing filters or closing-opening filters. In order to make the image grey values unchanged while filters can filter out nolse well, some scholar proposed morphological hybrid filters; ombine morphological opening-closing filters and morphological closing-opening filters or combine generalized morphological openingclosing filters and generalized morphological closing-opening filters to averaging, so that we can get basic morphogical hybrid filters and generalized morphological hybrid filters. The definitions of the two kinds of morphological hybrid filters are (we use $\mathrm{H}$ as hybrid):

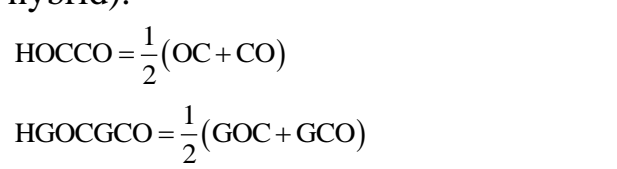

We can know from $[17]$ that these two kinds of morphological hybrid filters are good at suppressing noise, compensating grey values deviation of images and consuming shorter time.

The paper propose a better kind of morphological hybrid filter through expanding above-mentioned two kinds of hybrid filters, that is comprehensive morphological hybrid filter considering maintaining symmetry(Comprehensive Morphological Hybrid Filter, we use CMH to describe it).

\section{Comprehensive Morphological Hybrid Filter Considering Maintaining Symmetry}

The purpose of maintaining symmetry is to make new morphological filters maintain morphological hybrid filters' existed advantages, that is this filter does not depend on whether started with opening filter or closing filter, and it has the dual advantages of the two-path dual filter, it can also make the image grey values unchanged while filter out noise well. 
Definition 1 Based on basic morphological opening-closing filters, closing-opening filters and generalized morphological opening-closing filters, closing-opening filters, through maintaining symmetry comprehensively, we can get comprehensive morphological hybrid filter considering maintaining symmetry:

$\mathrm{CMH}=\omega_{1}\left\llcorner\mathrm{OC}+\omega_{2} \_\mathrm{CO}+\omega_{3} \sqsubset \mathrm{GOC}+\omega_{4} \_\mathrm{GCO}\right.$

$$
=\omega_{1} \square\left(f \circ k_{1} \bullet k_{1}\right)+\omega_{2} \square\left(f \bullet k_{1} \circ k_{1}\right)+\omega_{3} \square\left(f \circ k_{2} \bullet k_{3}\right)+\omega_{4} \square\left(f \bullet k_{2} \circ k_{3}\right)
$$

$k_{1}, k_{2}, k_{3}$ are three structural elements, they meet $k_{1}=k_{2}$ or $k_{1} \neq k_{2}$ but $k_{2} \neq k_{3}$ and $k_{2} \subseteq k_{3}$, the weight satisfies $\sum_{i=1}^{4} \omega_{i}=1, \omega_{1}=\omega_{2}$ and $\omega_{3}=\omega_{4}$.

The filter's founded conditions are to maintain two-path dual filters' symmetry from above definitions, which is the importance of morphological hybrid filter operator.

Theorem 1 The morphological hybrid filters not only reserved double filtering ability of dual morphological filters but also eliminated images' deviation after being filtered [17].

The proof of comprehensive morphological hybrid filter considering maintainng symmetry also satisfy this property is as follows.

Proof Define $f$ as images being polluted by Gaussian noise and define $g$ as original images, supposing that filters can filter out noise completely, so:

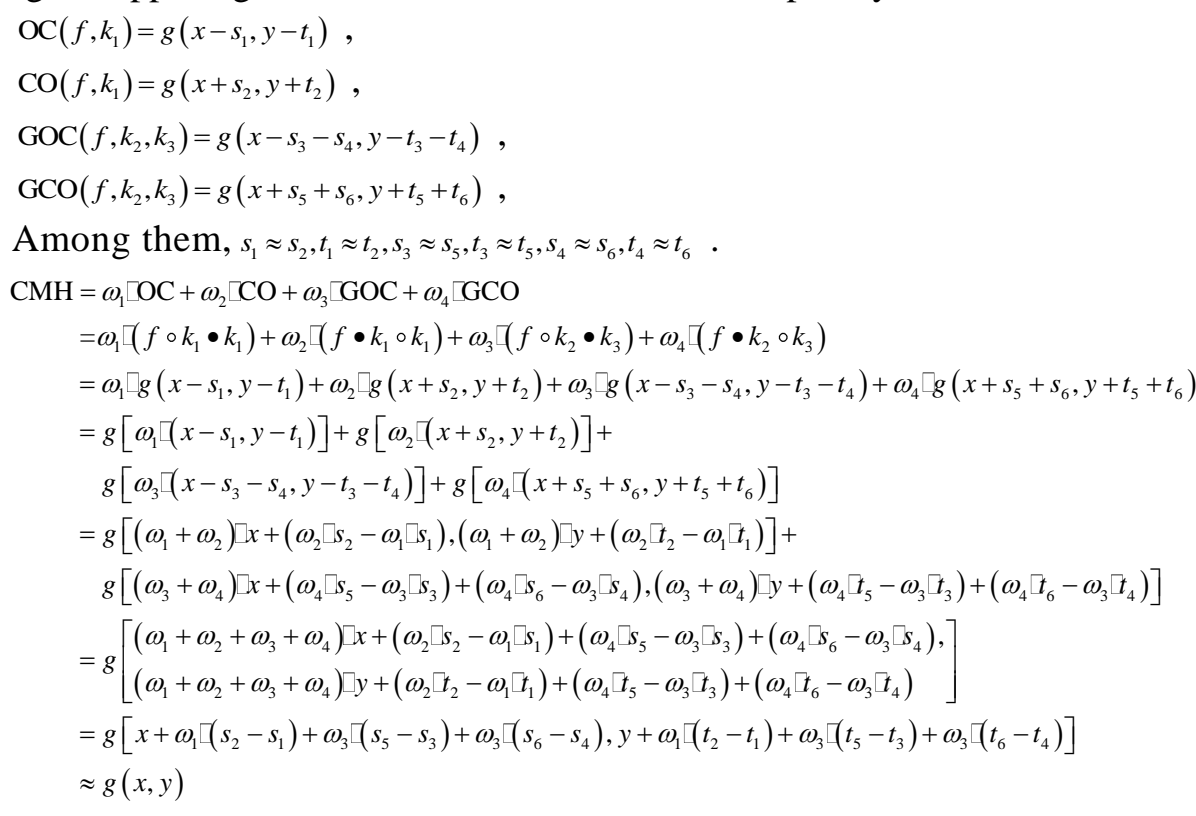

Thus, comprehensive morphological hybrid filter considering maintaining symmetry also has the ability of double filtering and eliminate the grey values deviation of images.

Because horphological opening-closing filters, closing-opening filters and generalized morphological opening-closing filters, closing-opening filters all satisfy translation invariance incremental property and duality[11,16], it is not difficult to prove that comprehensive morphological hybrid filter considering maintaining symmetry also satisfy these properties, that is:

(1) Translation invariance

$\mathrm{CMH}[f(x-s, y-t)+m]=\mathrm{CMH}[f(x-s, y-t)]+m$

Among them, $s, t \in Z^{2}, m \in R, Z, R$ is the integer set, the real number set respectively.

(2) Incremental property

If $f_{1}(x, y) \leqslant f_{2}(x, y)$,

$\mathrm{CMH}\left[f_{1}(x, y)\right] \leqslant \mathrm{CMH}\left[f_{2}(x, y)\right]$

(3) Duality

$$
[\mathrm{CMH}(f(x, y))]^{c} \leqslant \mathrm{CMH}\left[f^{c}(x, y)\right]
$$


We use symbol to represent complement or remainder operation.

Those three properties take advantage of the definition of comprehensive morphological hybrid filters considering maintaining symmetry and the properties of expansion, erosion, opening operation and closing operation. It's easy to prove it, so we ellipsis it.

The filtering process of this filter is as shown as Figure 1.

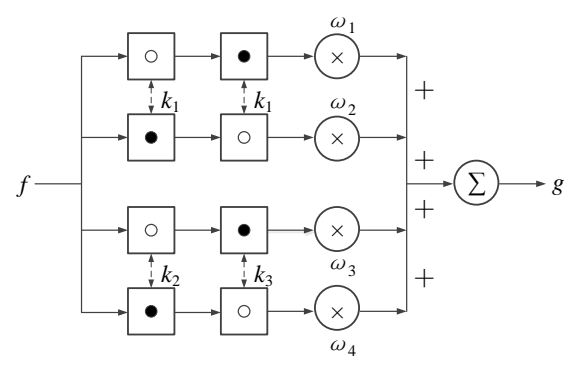

Figure 1. The Filtering Process of $\mathrm{CMH}$

\section{Simulation Experiment and Result Analysis}

Using "Lena" 512*512 pixels image to prove the effectiveness of filtering algorithm. Using comprehensive morphological hybrid filters considering maintaining symmetry to deal with images added with Salt \& Pepper moise(Salt \& Pepper),Gaussian noise(Gaussian) and mixture of that t 0 kinds in different intensity, and using morphological opening-closing, closing-opening Riltering method, generalized morphological opening-closing, closing-opening filterng method, morphological hybrid and generalized morphological hybry filtering method to deal with the same noise image. Finally we will compare the filtering results of these seven kinds of filtering method. The basic morphological filtering method(OC, CO and HOCCO) make use of structural elements with a square of $3 \times 3$ the generalized morphological filtering method(GOC, GCO, $\mathrm{HGOCGCO}_{3} \mathrm{CMH}$ ) make use of structural elements with a square of $3 \times 3$ and a square of $5 \times 5$, the filter's weight of CMH is $\omega_{1}=\omega_{2}=0.2$ and $\omega_{3}=\omega_{4}=0.3$.

We use image's MSE, PSNR and runtime as measurable indicators to evaluate and analysis filtering results. Supposing that there are images $A(x, y)$ and $B(x, y)$, so their MSEs are:

$$
\begin{aligned}
& M S E=\frac{1}{m n} \sum_{x=0}^{m-1} \sum_{y=0}^{n-1}[A(x, y)-B(y, y)]^{2} \\
& P S N R=10 \lg \frac{255^{2}}{M S E}
\end{aligned}
$$

Figure 2 is the result analyzed by every kind of morphological filter of Salt \& Pepper noise inage that its noise density is 0.03 . As we can see, every kind of filter can filter out noise well in this noise density, but the filter proposed by the paper has the best ability of mantaining image details and eliminating grey values deviation of images among these three kinds of hybrid filters. Figure 3 is the result of MSE, PSNR and runtime of Salt \& Pepper noise images in different intensity analyzed by every kind of morphological filter. We can see from the PSNR resulting figure that the PSNR of $\mathrm{CMH}, \mathrm{OC}$ and $\mathrm{CO}$ is almost same when noise intensity is less than 0.03.But the ability of filtering of OC and CO weakened with the increase of noise intensity, the PSNR of CMH higher than GOC, GCO about $2 \mathrm{~dB}$ in this process, it shows that $\mathrm{CMH}^{\prime}$ ability of filtering out noise is better than the latter two. The PSNRs of those five kinds of filters all higher than other two kinds of hybrid filters: HOCCO and HGOCGCO, it shows that the ability of filtering out Salt \& Pepper noise of these two kinds of hybrid filters is weaker than the former five. This 
conclusion can be proved from MSE resulting figure. The runtime of this two kinds of hybrid filters is shorter than other filters, but the runtime of $\mathrm{CMH}$ is only $0.5 \mathrm{~ms}$.

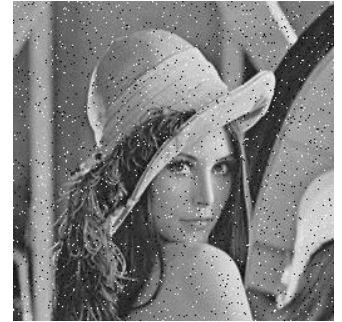

(1)Salt \& Pepper Noise

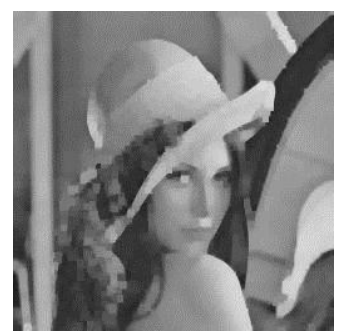

(5)GCO

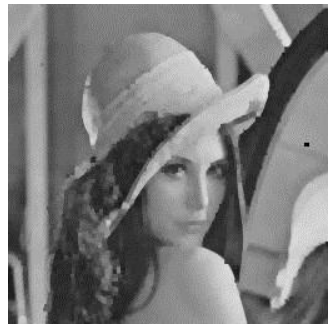

(2)OC

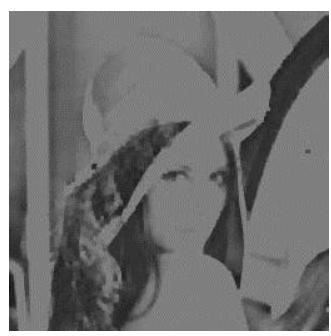

(6) HOCCO

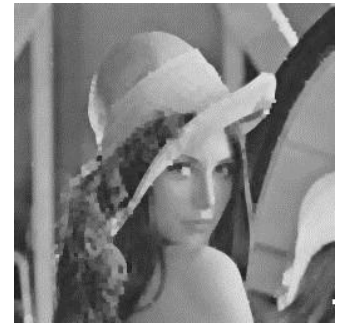

(3) $\mathrm{CO}$

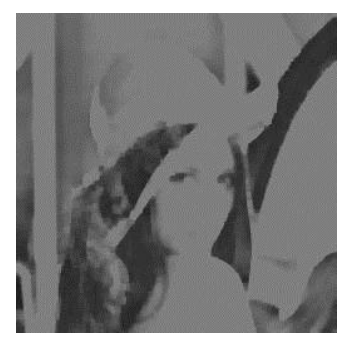

(7) HGOCGCO

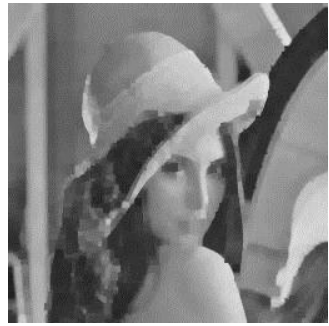

(4)GOC

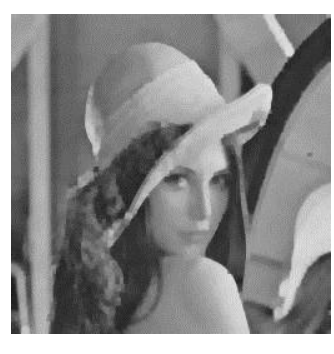

(8) $\mathrm{CMH}$

Figure 2. The Result of Salt \& Pepper Noise Îmages Analyzed by every Kind of Môrrohological Filter

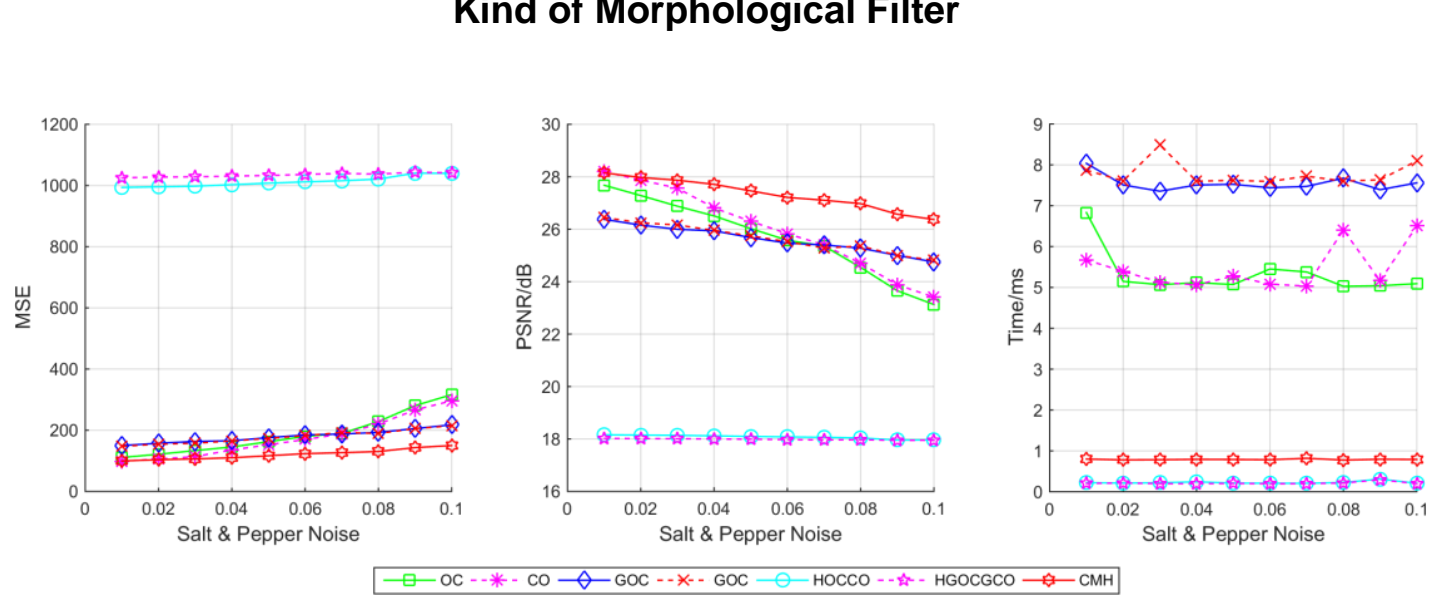

Figure 3. The Results of MSE, PSNR and Runtime of Salt \& Pepper Noise Images in Different Intensity analyzed by Every Kind of Morphological Filter

As we can see from Figure 2 and Figure 3,for Salt \& Pepper noise images, the niorphological hybrid filter's ability of filtering may not better than the morphological non-hybrid filter's. 


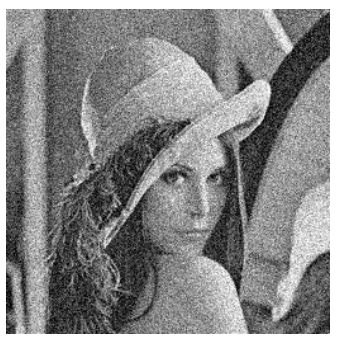

(1)Gaussian Noise

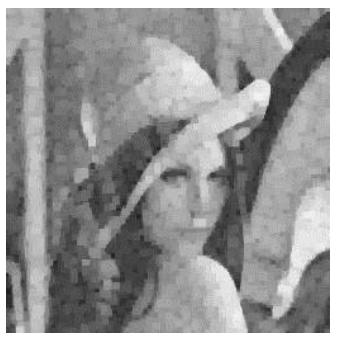

(5)GCO

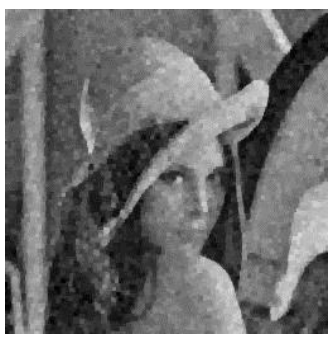

$(2) \mathrm{OC}$

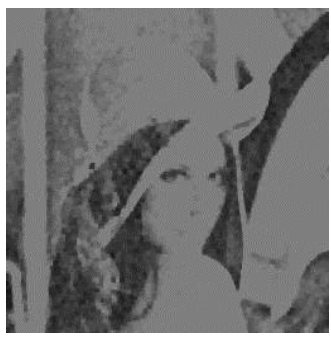

(6) HOCCO

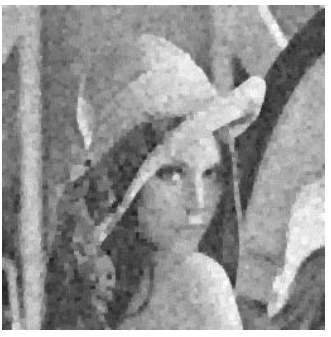

(3) $\mathrm{CO}$

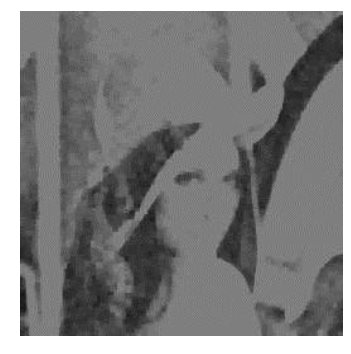

(7)HGOCGCO

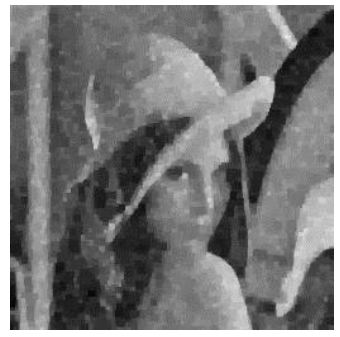

(4)GOC

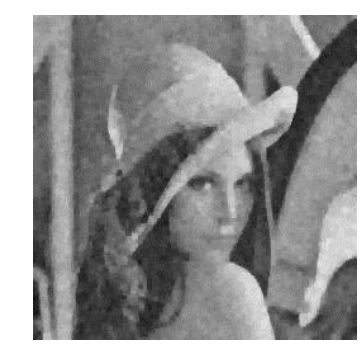

(8) $\mathrm{CMH}$

Figure 4. The Result of Gaussian Noise Images Analyzed by Every Kind of Morphological Filter

ological

Figure 4 is the result analyzed by every kind of morphological filter of Gaussian noise image that its mean is 0 ,variance is 0.01 . We can see from the figure that $\mathrm{CMH}$ is better than any other filters not only in the ability of filtermg but also in maintaining image details. Figure 5 is the result analyzed by every kind of morphological filter of MSE, PSNR and runtime. We can see from the MSE and PSNR resulting figure that these three kinds of morphological hybrid filters' ability of filtering are better than morphological non-hybrid filters' when roise intensity is more than 0.03 , and the runtime of these three kinds of morphological hybrid filters is less from the runtime resulting figure. Thus, morphological hybrid filters have better ability of filtering out Gaussian noise than morphological non-hybrid filters oyerall.
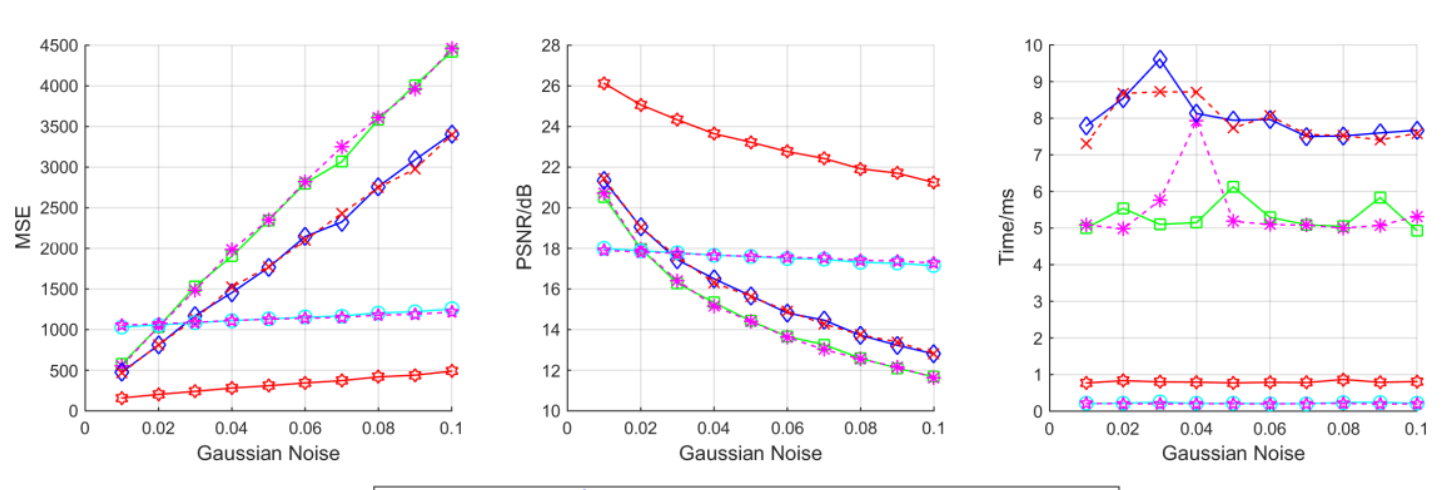

Figure 5. The Results of MSE, PSNR and Runtime of Gaussian Noise Images in Different Intensity analyzed by Every Kind of Morphological Filter

Figure 6 is the result analyzed by every kind of morphological filter of Salt \& Pepper noise image that its noise density is 0.03 , Gaussian noise image that its mean is 0 ,variance is 0.01 .As we can see from the figure, $\mathrm{CMH}$ has better abilities of dealing with hybrid noise and maintaining image details than other filters. Figure 7 is the result analyzed by every kind of morphological filter of MSE, PSNR and runtime. It can be seen from these 
three figures, the result of hybrid noise image analyzed by every kind of filter is similar to the result of Gaussian noise image, which is not affected by the weak ability of filtering out Salt \& Pepper noise and the strong ability of filtering out Gaussian noise. It shows that the morphological hybrid filters have better ability of filtering out complicated noise than the morphological non-hybrid filters.

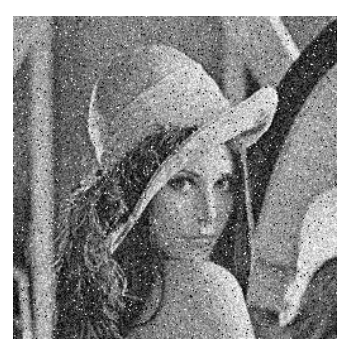

(1)Mixed Noise

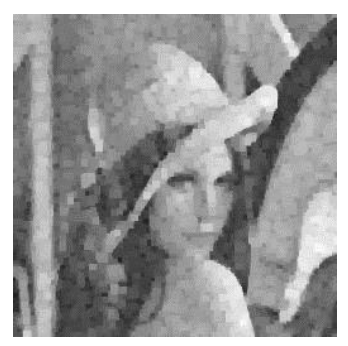

(5)GCO

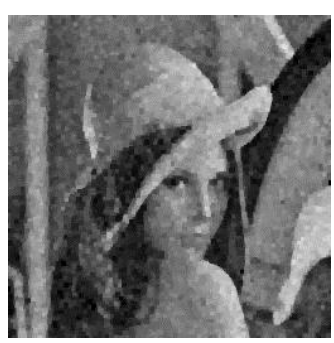

$(2) \mathrm{OC}$

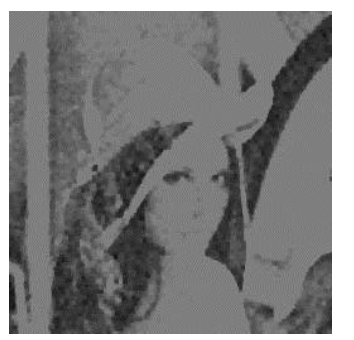

(6) HOCCO

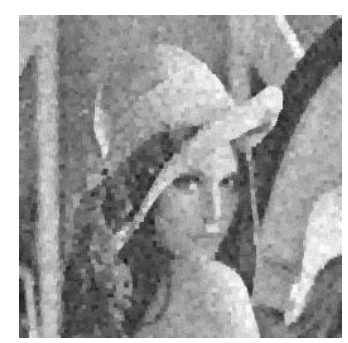

(3) $\mathrm{CO}$

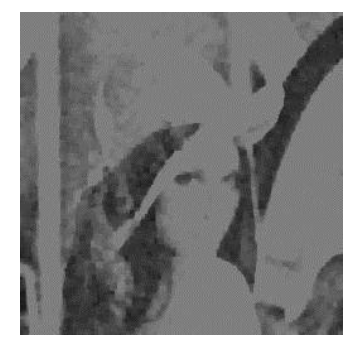

(7)HGOCGCO

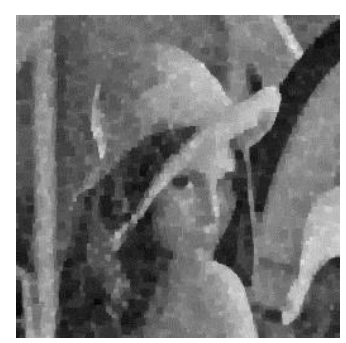

$(4) G O C$

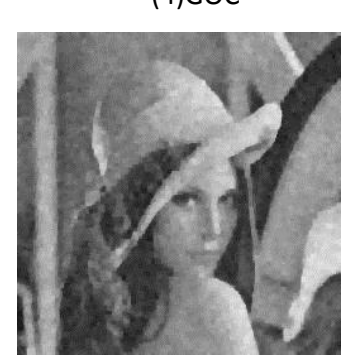

(8) $\mathrm{CMH}$

Figure 6. The Result of Mixed Noise Images Analyzed by Every Kind of Morphological Filter

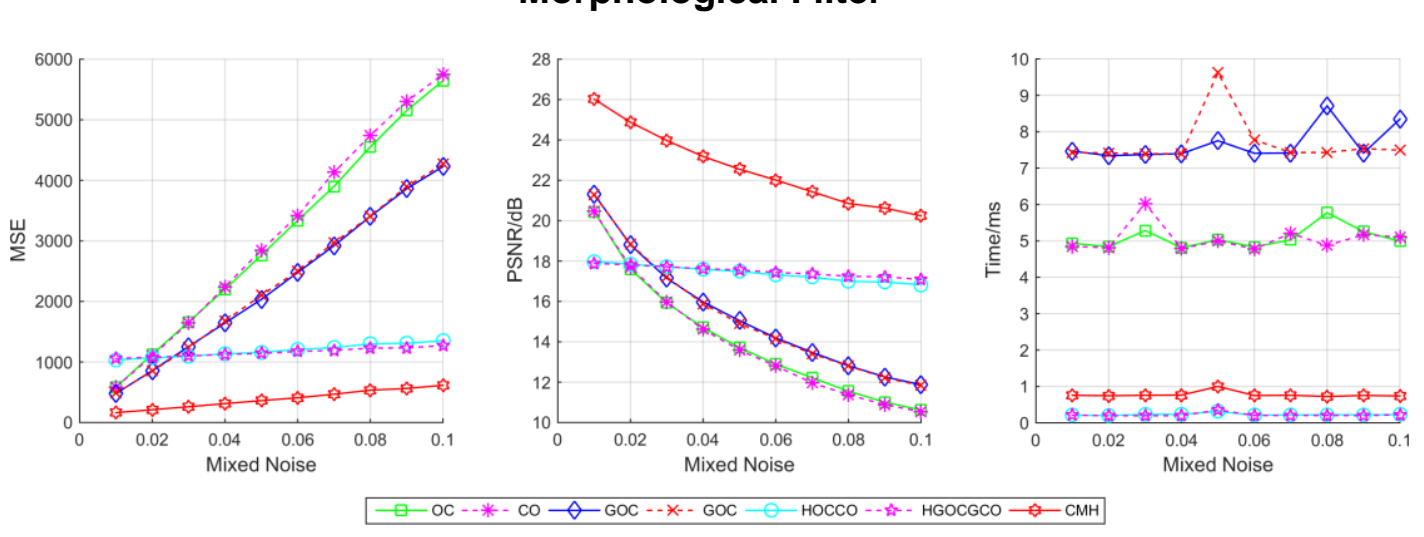

Figure 7 The Results of MSE, PSNR and Runtime of Hybrid Noise Images in Different Intensity Analyzed by every Kind of Morphological Filter

Figure 8 is the result analyzed by every kind of morphological filter of three kinds of nowe image's grey values deviation, and the left image is Gaussian noise, the middle image is Salt \& Pepper noise, the right image is hybrid noise. We select one of the columns of image signals, 50 consecutive pixels grey value at some point. We define Original as original image with no noise, and define Noise as image with noise. We can see from these three images that the hybrid filter considering maintaining symmetry have better ability of compensating grey values deviation of images after being filtered. And the filtered signal of $\mathrm{CMH}$ filter is similar to that of original image, it shows that this filter has better ability of compensating grey values deviation of images than other two kinds of hybrid filters. 

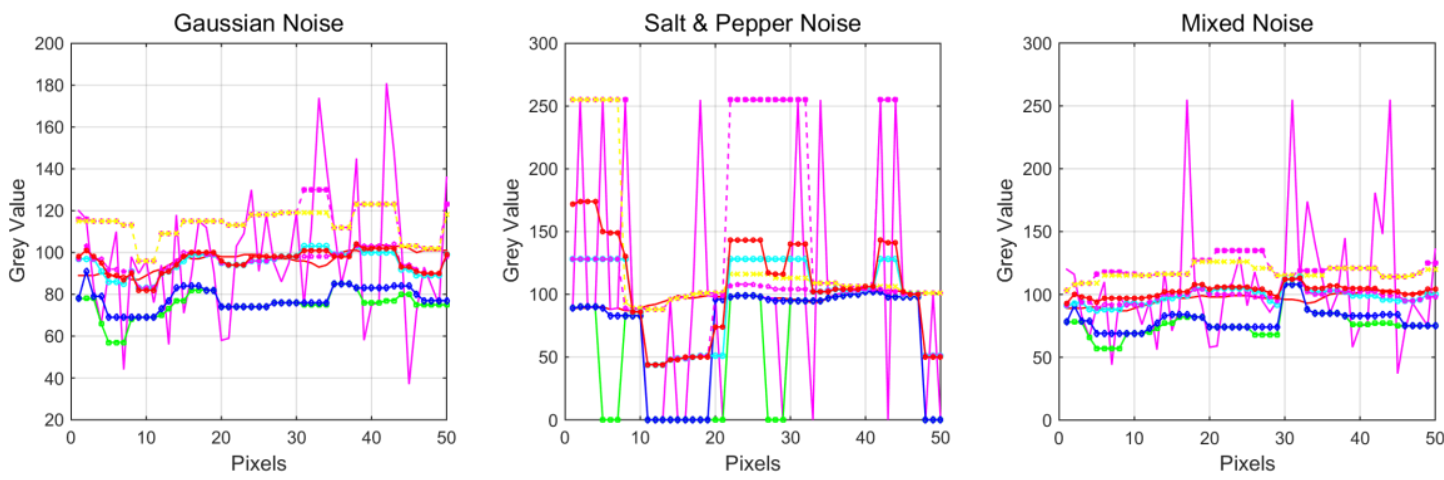

Figure 8. The Results of Grey Values Deviation of Noise Images in Different Intensity Analyzed by Every Kind of Morphological Filter
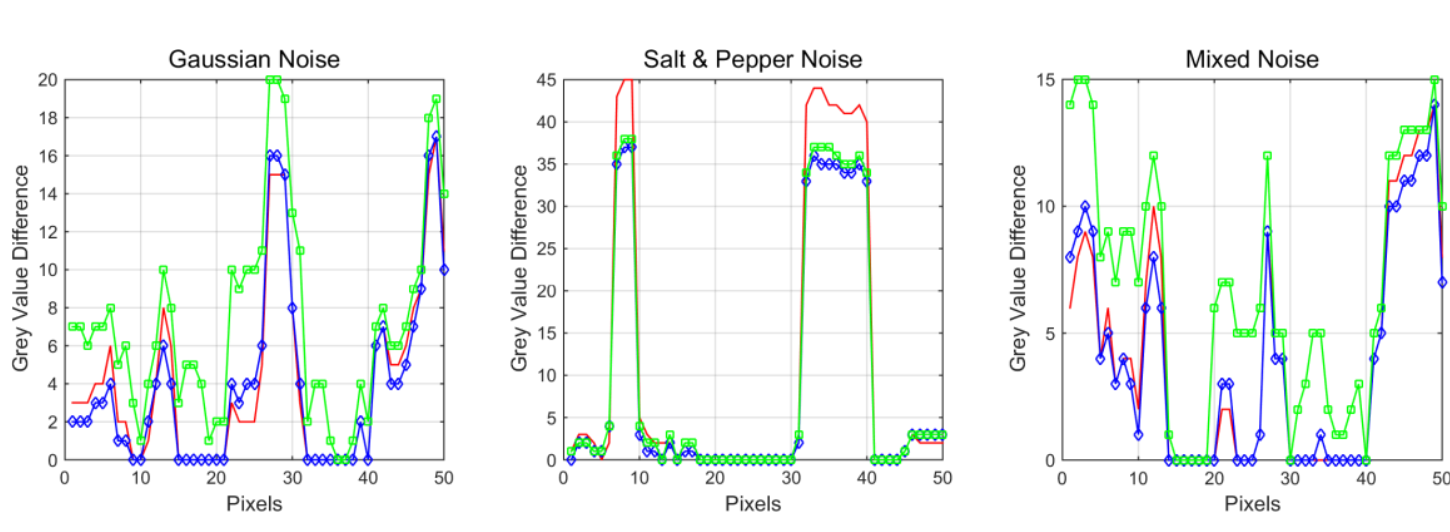

Figure 9. The Result of Grey Value Difference by CMH Filter Considering Maintaining Symmetry and Not

Figure 9 is the difference of noise lmages being filtered and original grey value images filtered by $\mathrm{CMH}$ filter when in considering maintaining symmetry and not considering maintaining symmetry. The left image is Gaussian noise, the middle image is Salt \& Pepper noise, and the right mage is hybrid noise. We select one of the columns of image signals, 50 consecutive pixels grey value at some point. We can see that hybrid filter's ability of maintaining ymmetry has direct influence on the ability of compensating grey values deviation of images.

We can know that the morphological hybrid filters are good in filtering out complicated noise, but $\mathrm{CMH}$ has better abilities of filtering out Salt \& Pepper noise, Gaussian noise and mixed noise than other six kinds of existing morphological filters. It shows that $\mathrm{CMH}$ is effective.

\section{Conclusions}

The paper analyzes basic morphological opening-closing, closing-opening filters, generalized morphological opening-closing, closing-opening filters and morphological hybrid filters. Based on this, we propose comprehensive morphological hybrid filters considering maintaining symmetry. Through simulation experiment and comparing filtering results with other morphological filters, we prove that $\mathrm{CMH}$ has better abilities of filtering and maintaining image details than other morphological filters, and runtime is shorter. And we can learn from the experiment that the filter considering maintaining 
symmetry the paper proposed have better ability of compensating grey values deviation of images than those filters not considering maintaining symmetry.

\section{Acknowledgement}

This work is supported by the National Natural Science Foundation of China (No. 41501425, No. 41561084), and Shandong Natural Science Foundation of China (No. ZR2014DL001).

\section{References}

[1] Tang Changqing, Mathematical morphology method and its application, Science press, Beijing (1990)

[2] Duan Shan, Mathematical Morphology and its Application Research in Remote Sensing Image Processing, Wuhan University, Wuhan (2004)

[3] Liu Sheng, Wang Xiaoyu, Qiu Xinfa, Meteorology and Disaster Reduction Research. A mathematical morphology filtering algorithm for high-resolution remote sensing Image. 31, 4(2008)

[4] Lei Tao, Fan Yangyu, Mao Li, Journal of Optoelectronics Laser. Generalized self-dual molphological filters and the applications in image-denoising. 21, 1 (2011)

[5] Chen Hu, Wang Shouzun, Zhou Chaohui, Journal of Engineering Graphics. Research based on mathematics morphology image edge examination method. 2 (2004)

[6] Lin H, Du P J, Shu N, Remote Sensing Technology and Application .Edge detection method of remote sensing images based on mathematical morphology of multi-S ructural elements. 19, 2(2004)

[7] Wang Huifeng, Zhan Guili, Lu Xiaoming, Computer Engineering and Applications. Research and application of edge detection operator based on mathematical morphology. 31, 2(2009)

[8] Tang Yonghe, Lu HuanZhang, Geomatics and Information Science of Wuhan University, Morphological Edge Detection Algorithm Based on Multi-structure Elements Compound Filter, (2012)

[9] An R, Feng X Z, Wang H L, Journal of Image and Graphics, Road feature extraction from remote sensing classified imagery based on mathematical morphology and analysis of road networks. 8, 7 (2003)

[10] Pan Jianping, Li Zhi, Computer Engineering and Applications .Road Extraction based on Multistructure Element of Mathematical Mophology. 46, 11(2010)

[11] Bai Yingan, Yu Shentlin, Li Jianming, Journal of Image and Graphics .A new generalized open and close morphological filters. 14, 8(2009)

[12] Ren Huorong, Mathematical Morphology and Application, Xidian University, Xi'an (2004)

[13] [13] Haralick R M, Stemberg S R, Zhuang X, IEEE transactions on pattern analysis and machine intelligence .Image analysis-using mathematical morphology. 9, 4(1987)

[14] [14] Serra J, Signal processing. Morphological filtering: an overview. 38, 1(1994)

[15] [15] Song J, DELP E J, Chlcaits, Systems, and Signal Processing. A study of the generalized morphological filter. 11, 1(1992)

[16] [16] Zhao Chunhui, Sun Shenghe, Journal of Electronics .Generalized parallel complex morphological filters with multiple structurng elements. 20, 1(1998)

[17] [17] Lei Tao, Fan Kangyu, Bai Bo. Acta Automatica Sinica. A New Class of Generalized Morphological Hybrid Filters. 37, 2(2011)

[18] [18] Mingyue Zhao, Dongxue Zhao, Zhixia Jiang, Diaomei Cui, Jie Li and Xingyi Shi , Mathematical modelling of engineering probolems. The gary prediction GM $(1,1)$ model in traffic forecast. 2, 1(2015)

[19] [19] Tu Jihui, Review of computer engineering studies. A novel building boundary extraction method for high-resolution aerial image.1, 2(2014)

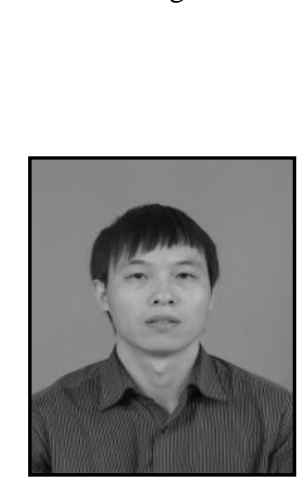

\section{Authors}

Yuefeng Lu, He received the Bachelor of Science degree in computer sciences from Henan Normal University, Xinxiang, China, in 2005, and the Ph.D. degree in cartography and geographic information engineering from Wuhan University, Wuhan, China, in 2013, after a six-year joined study of Master and Doctoral Degree. From 2005 to 2007 he was a teaching assistant of computer sciences at Sanmenxia Polytechnic, Sanmenxia, China. His professional interests lie in image processing, geographical spatial data matching, and geographic information system theory and its engineering applications. 